\title{
Tratamento da síndrome da dor patelofemoral com treinamento neuromuscular:
}

\section{Uma breve revisão}

\author{
Treatment of patellofemoral pain syndrome with neuromuscular training: A brief review \\ Tratamiento del síndrome de dolor femororrotuliano con entrenamiento neuromuscular: Una
}

breve revisión

Recebido: 07/07/2021 | Revisado: 13/07/2021 | Aceito: 14/07/2021 | Publicado: 24/07/2021

Givanildo de Oliveira Santos

ORCID: https://orcid.org/0000-0001-5279-1535

Faculdade de Mauá Brasília, Brasil

E-mail: givanildo-o@hotmail.com

Wilker Almeida Cruz

ORCID: https://orcid.org/0000-0002-4029-2269

Faculdade de Piracanjuba, Brasil

E-mail: wilkercruzprof@gmail.com

Thais Nunes Couto

ORCID: https://orcid.org/0000-0002-7350-6550

Faculdade de Piracanjuba, Brasil

E-mail: thaisnunesufg@gmail.com

Raquel Borges de Souza

ORCID: https://orcid.org/0000-0003-1675-6649

Faculdade de Piracanjuba, Brasil

E-mail: raquelsab1980@gmail.com

Sebastião Lobo da Silva

ORCID: https://orcid.org/0000-0003-2639-0898

Universidade Católica de Brasília, Brasil

E-mail: slobo2011@gmail.com

\begin{abstract}
Resumo
A síndrome da dor patelofemoral é uma patologia que acarreta dor e desconforto na região posterior da patela, comprometendo a força, estabilidade e funcionalidade da articulação do joelho afetando a realização de tarefas simples do dia a dia em adultos jovens e comprometendo o desempenho em atletas independente do sexo. Com vasta literatura, mas ainda várias lacunas sobre formas de tratamento que seriam mais eficazes na melhora da dor, da funcionalidade, estabilidade e aumento de força. Objetivou-se identificar na literatura o tipo de tratamento mais eficaz na melhora da dor e funcionalidade do indivíduo diagnosticado com a síndrome patelofemoral. Este estudo realizou uma revisão de literatura sobre estudos referentes ao tratamento com base em exercício e fortalecimento neuromuscular. A busca foi realizada em base de dados eletrônica. A busca eletrônica resultou em 150 periódicos, após a análise dos mesmos, 10 estudos preencheram os critérios de inclusão definido, sendo adicionados mais 17 artigos sobre a patologia e anatomia do joelho. Este estudo concluiu que os protocolos de tratamento que envolveram exercícios voltados para o fortalecimento associados a exercícios de fortalecimento do quadríceps, demonstraram maior eficácia quando comparados individualmente, também concluindo que os indivíduos que se mantiveram ativos após as intervenções não voltaram a sentir dor.
\end{abstract}

Palavras-chave: Treinamento resistido; Anatomia; Doenças; Ligamentos; Músculos.

\begin{abstract}
Patellofemoral pain syndrome is a pathology that causes pain and discomfort in the posterior region of the patella, compromising the strength, stability and functionality of the knee joint, affecting the performance of simple daily tasks in young adults and compromising performance in independent athletes of sex. With extensive literature, but still several gaps on forms of treatment that would be more effective in improving pain, functionality, stability and increased strength. The objective was to identify in the literature the type of treatment most effective in improving pain and functionality in the individual diagnosed with patellofemoral syndrome. This study carried out a literature review on studies related to treatment based on exercise and neuromuscular strengthening. The search was carried out in an electronic database. The electronic search resulted in 150 journals, after analyzing them, 10 studies met the defined inclusion criteria, adding 17 more articles on the pathology and anatomy of the knee. This study concluded that treatment protocols that involved strengthening exercises associated with quadriceps strengthening exercises, demonstrated greater effectiveness when compared individually, also concluding that individuals who remained active after the interventions did not experience pain again.
\end{abstract}

Keywords: Resistance training; Anatomy; Illnesses; Ligaments; Muscles. 


\begin{abstract}
Resumen
El síndrome de dolor femororrotuliano es una patología que provoca dolor y malestar en la región posterior de la rótula, comprometiendo la fuerza, estabilidad y funcionalidad de la articulación de la rodilla, afectando el desempeño de tareas cotidianas sencillas en adultos jóvenes y comprometiendo el rendimiento en deportistas independientes de sexo. Con literatura extensa, pero aún con varias lagunas sobre formas de tratamiento que serían más efectivas para mejorar el dolor, la funcionalidad, la estabilidad y el aumento de la fuerza. El objetivo fue identificar en la literatura el tipo de tratamiento más efectivo para mejorar el dolor y la funcionalidad en el individuo diagnosticado de síndrome femororrotuliano. En este estudio se realizó una revisión de la literatura sobre estudios relacionados con el tratamiento basado en el ejercicio y el fortalecimiento neuromuscular. La búsqueda se realizó en una base de datos electrónica. La búsqueda electrónica resultó en 150 revistas, luego de analizarlas, 10 estudios cumplieron con los criterios de inclusión definidos, con 17 artículos adicionales sobre la patología y anatomía de la rodilla. Este estudio concluyó que los protocolos de tratamiento que involucraron ejercicios de fortalecimiento asociados con ejercicios de fortalecimiento de cuádriceps, demostraron mayor efectividad cuando se compararon individualmente, también concluyendo que los individuos que permanecieron activos luego de las intervenciones no volvieron a experimentar dolor.
\end{abstract}

Palabras clave: Entrenamiento de resistencia; Anatomía; Enfermedades; Ligamentos; Músculos.

\title{
1. Introdução
}

Dentre os problemas mais acometidos quando se trata de articulação de joelho, uma das patologias mais frequentes é a dor patelofemoral, também conhecida como síndrome da dor femoropatelar. Esta disfunção, geralmente ocorre em adolescentes fisicamente ativos, corredores amadores, profissionais e mulheres, incomodando muito ao realizar tarefas do dia a dia como subir e descer escadas, realizar agachamentos e saltos, ou seja, movimento que se usa para realizar pequenas tarefas do cotidiano (Dye, 2005).

A patela é um osso do tipo sesamóide, está localizada dentro da estrutura do tendão do quadríceps, sendo sua cartilagem a mais espessa do corpo medindo entre 4 e $6 \mathrm{~mm}$ em adultos jovens saudáveis. Responsável pelo aumento de força do quadríceps com o percentual de força equivalente entre 33 e 50\%, atua como estabilizador estático, é uma articulação que em fase de movimento sua área de contato com a tróclea muda ao longo da magnitude do mesmo (Gaitonde, et al., 2019).

Quanto à estabilidade patelar, ela é dependente de restrições de tecidos moles localizados na face anterior do joelho. O tendão patelar, tendão do quadríceps e os retináculos medial e lateral atuam como estabilizadores apoiados pelo músculo vasto medial oblíquo limitando o mal rastreamento quando a flexão do joelho se perpetua entre 0 e $30^{\circ}$ (Gaitonde, et al., 2019).

As causas do acometimento da articulação do joelho podem ser variadas, dentre elas estão: desalinhamento patelofemoral, desequilíbrio dos tecidos periarticulares moles, fraqueza muscular do quadríceps, desequilíbrio muscular entre o vasto lateral e medial e anormalidades ósseas, ou seja, impossível diagnosticar uma única causa (Gulati, et al., 2018).

Entre as formas de tratamento, uma delas é o fortalecimento muscular na região do quadríceps e quadril juntamente com exercícios de alongamento e correção postural (Saltychev, et al., 2018). Conforme este cenário, este estudo, objetivou identificar na literatura o tipo de tratamento mais eficaz na melhora da dor e funcionalidade do indivíduo diagnosticado com a síndrome patelofemoral.

\section{Metodologia}

De acordo com Estrela (2018), a presente pesquisa trata-se de uma revisão bibliográfica de natureza qualitativa, para o levantamento bibliográfico foram utilizados artigos científicos disponíveis online. As buscas dos artigos foram realizadas nas plataformas de pesquisas: PubMed, Lilacs, Google acadêmico, MedLine, ScienceDirect, SciELO, ScienceDirect e Elsevier, no período de agosto/2020 a janeiro/2021. Para as buscas foram usadas palavras-chave na língua inglesa sendo elas Patellofemoral pain syndrome; treatment e anatomical structures of the knee, identificando 150 resultados, sendo selecionados 27 estudos de acordo com a temática deste estudo, não havendo limite de data para os estudos usados para revisão de literatura.

Destes foram escolhidos 10 artigos para compor a análise de resultados, com os seguintes critérios de inclusão, ambos 
deveriam ter amostra humana, independente de sexo e idade e deveriam ser diagnosticados com a patologia síndrome da dor patelofemoral, as amostras deveriam ser sujeitas há formas de tratamento que envolvam exercício físico para melhora da dor e funcionalidade, os estudos deveriam ser originais, optou-se por não incluir teses e dissertações.

\section{Revisão}

\subsection{Síndrome da dor patelofemoral (PFPS) ou Dor patelofemoral (PFP)}

A síndrome da dor patelofemoral é uma das causas mais comuns de dor anterior do joelho encontrada no ambiente ambulatorial em adolescentes e adultos com menos de 60 anos (Dye, 2005). A nomenclatura desta enfermidade varia de estudo para estudo, nas referências citadas, encontrou-se a nomenclatura sendo de duas formas: Síndrome da dor patelofemoral (PFPS) ou Dor patelofemoral (PFP).

A incidência nos Estados Unidos está entre 3\% e 6\%, a característica principal do PFPS é a dor na região anterior do joelho, que se intensifica quando o joelho é flexionado durante as atividades de levantamento de peso. A dor da PFPS, geralmente piora quando o indivíduo permanece sentado por período prolongado ou quando desce de escadas (Zamboti, et al., 2017).

Um exame físico mais perceptível será encontrado no paciente quando o mesmo sente dor ao agachar, examinando a forma como o paciente anda, a postura e os sapatos podem ajudar a identificar as causas contribuintes. Radiografias simples do joelho não são necessárias para o diagnóstico de PFP, mas podem excluir outros diagnósticos, como osteoartrite, fratura patelar e osteocondrite (Glaviano, et al., 2015).

O tratamento da PFPS inclui repouso, um curto uso de anti-inflamatórios não esteroidais e fisioterapia direcionada ao fortalecimento dos músculos flexores do quadril e joelho. A bandagem patelar kinesio pode fornecer alívio adicional da dor de curto prazo; entretanto, as evidências são insuficientes para apoiar seu uso rotineiro (Zamboti, et al., 2017).

A principal característica do PFPS é a dor no joelho, ou ao redor do joelho anterior que se intensifica quando o joelho é flexionado durante as atividades de levantamento de peso. Os pacientes também podem sentir dor ou rigidez exacerbada por ficar sentado por muito tempo com o joelho em flexão. A PFPS é uma forma comum de lesão por uso excessivo do joelho (Piazza, 2012).

Os pacientes devem ser questionados sobre lesões e cirurgias anteriores no joelho, nível de atividade atual e mudanças recentes na atividade. Menos comum, os pacientes podem sentir dor ao executarem a flexão do joelho, que é devido à fraqueza no quadríceps, resultando em breve perda de tônus muscular, e não instabilidade da articulação intrínseca do joelho (Dye, 2005).

Uma declaração de Consenso de 2016 define PFPS como uma dor que ocorre ao redor ou atrás da patela que é agravada por pelo menos uma atividade que carrega a patela durante a sustentação de peso em um joelho flexionado, atividades contribuintes incluem correr, subir escadas, pular e agachar, como também é conhecida de síndrome da dor no joelho do corredor e na região anterior do joelho. Embora o termo PFPS tenha sido usado de forma intercambiável com condromalácia patelar, o último, refere-se especificamente ao achado de cartilagem patelofemoral amolecida em radiografia simples, ressonância magnética ou artroscopia de joelho (Gaitonde, et al., 2019).

Devido aos múltiplos fatores que contribuem para a PFPS, a terapia deve ser individualizada, os movimentos ou atividades exacerbadas devem ser evitados, embora o paciente deva permanecer o mais ativo possível (Piazza, 2012).

Alguns estudos concluíram que os pacientes que se exercitam, geralmente apresentam melhora na dor de curto e longo prazo, tanto em repouso quanto com atividade, quando comparados com aqueles que não praticam exercícios devem se concentrar no quadril e joelho. Não existem dados de alta qualidade para recomendar um tipo de exercício em detrimento de outro. O fortalecimento dos músculos que envolvem o joelho reduz a pressão na articulação patelofemoral ao estabilizar o 
recrutamento muscular (Gaitonde, et al., 2019).

\subsection{Anatomia do joelho}

O joelho é composto por duas articulações, sendo uma articulação tíbio-femoral e uma patelofemoral. A articulação tíbio-femoral é o lugar que ocorre a transmissão do peso corporal do fêmur para a tíbia, proporcionando rotação articular no plano sagital juntamente com um pequeno grau de rotação. A articulação patelofemoral age como mecanismo extensor, juntamente com os músculos do quadríceps e tibial anterior trabalhando em conjunto com a articulação do tornozelo para dissipar a tensão causada pelo impacto quando o corpo entra em fase de apoio no ciclo da marcha quando vai para frente (Amis, 2007).

A patela é o maior osso sesamoide do corpo, é revestida pela camada retinacular do mecanismo extensor, essa camada se insere na camada mais profunda proximamente ao tendão patelar e distal ao vasto intermédio. A crista central vertical que divide a faceta lateral mais ampla da faceta medial se localiza na superfície da patela. A patela se articula com o sulco femoral, este que por sua vez possui uma crista lateral mais alta que a porção medial em relação a patela atribuindo maior estabilidade óssea à articulação, quando a patela está envolvida em um ângulo de aproximadamente $45^{\circ}$ de flexão de joelho (Cohen, et al., 2008).

Os ligamentos capsulares são estruturados como retináculo medial, retináculo lateral e ligamentos do mecanismo extensor, prende-se distalmente à tíbia e na parte anterior dos meniscos, não possuindo anexos femorais organizados. O mecanismo extensor tendíneo é estruturado em três camadas, sendo a camada arciforme, camada retinacular e o ligamento iliopatelar. O tendão patelar é uma extensão do tendão quadriciptal, estrutura que se insere desde o polo inferior da patela até a tuberosidade anterior da tíbia, ele se estende desde o polo inferior da patela se inserindo no tubérculo medial sendo separado da tíbia pela Bursa do tendão infrapatelar (Flandry \& Hommel, 2011).

Basicamente as estruturas sinoviais são formadas pela plica e pela almofada de gordura infrapatelar. A plica sinovial do joelho é variável de indivíduo para indivíduo, sua formação pode variar de uma completa septação da bolsa suprapatelar a uma banda que pode se estender da camada de gordura medial através da bolsa suprapatelar. A almofada de gordura normalmente é constituída de tecido adiposo e uma arcada fornece suprimento vascular primário do ligamento cruzado anterior (Flandry \& Hommel, 2011).

A estabilização articular é dependente da interação de elementos estáticos e dinâmicos, a estabilidade dinâmica envolve músculos que agem na articulação do joelho como é o caso do quadríceps sendo o principal desacelerador do joelho. Já a estabilidade estática é derivada de ligamentos tibiofemorais, meniscos e cabeça dos gastrocnêmicos, pois é muita ingenuidade atribuir toda a estabilidade do joelho aos ligamentos tibiofemorais anterior (Flandry \& Hommel, 2011).

Possuem uma anatomia complexa responsável por diversas funções biomecânicas como suporte da carga, guiam a rotação e agem como estabilizadores. O menisco medial é mais largo, embora seja ligeiramente mais fino, sendo localizado na parte central, posteriormente a coluna intercondilar perto da fóvea central. O menisco lateral possui forma de pires devido ao formato da topografia convexa do planalto tibial lateral sendo semelhante a uma gravata borboleta (Makris, et al., 2011).

O ligamento cruzado anterior (LCA), origina-se na superfície póstero lateral do entalhe intercondilar inserindo na eminência intercondilar. Contêm 2 pacotes, um anteromedial e um feixe póstero lateral que sofrem vários graus de tensão à medida que flexiona e que a tíbia gira internamente. Seu suprimento de sangue acontece através de anastomose formada por artérias geniculares através da camada de gordura, por sua vez quando é rompido se torna uma lesão de recuperação extremamente complexa, pois esses vasos também se rompem, cortando, assim o suprimento sanguíneo anterior (Flandry \& Hommel, 2011).

Ligamento cruzado posterior (LCP), origina-se na superfície medial do entalhe intercondilar se estendendo 
distalmente e inserindo na fóvea central. Sua origem proximal acompanha o eixo epicondilar do fêmur representando o centro de rotação axial do joelho. Possui dois feixes, um póstero medial que é mais tensionado quando há extensão e um anterolateral mais tensionado quando há flexão. Ao contrário do LCA (Ligamento cruzado posterior), se caso for rompido, ele não perde o suprimento de sangue, tornando sua recuperação mais rápida (Arthur, et al., 2020).

\subsection{Intervenção para o tratamento da Síndrome da dor patelofemoral}

A síndrome da dor patelofemoral não possui causa específica e acomete vários tipos de público, como jovens ativos, atletas, mulheres. Dentro dessa realidade, uma das opções de tratamento referente ao sintoma de dor e funcionalidade é o fortalecimento muscular. Muito se fala sobre tipo de exercício que seria mais adequado, como exercício resistido ou alongamento e também qual grupamento muscular estes exercícios deveriam envolver.

Ferber, et al. (2015), dividiram a sua amostra em dois grupos, ambos realizaram exercícios focados na musculatura do quadríceps, com apenas uma diferença entre os grupos. No grupo 1 foram adicionados exercícios com foco no fortalecimento da musculatura que envolve o quadril, estes protocolos duraram a cerca de seis semanas. Identificaram que ambos os grupos obtiveram resultados positivos no ganho de força, melhora da funcionalidade e ausência de dor, sendo que o grupo que realizou exercícios de fortalecimento do quadríceps e do quadril obteve ausência de dor na terceira semana enquanto o grupo 2 que realizou somente exercícios para o quadríceps obtiveram a ausência de dor somente na quarta semana. Resultados semelhantes foram identificados por (Dolak, et al., 2011) e (Baldon, et al., 2014).

Outro fator intrigante em relação aos resultados era o tempo em que eles se mantinham em função do problema. Fukuda, et al., (2012), resolveram acompanhar cinquenta e quatro mulheres durante quatro semanas, sendo divididas em um grupo que realizava fortalecimento muscular do quadril e quadríceps e outro grupo que fortalecia apenas o quadríceps. Como resultado o grupo que realizava exercícios para o quadril e quadríceps tiveram melhora superior da funcionalidade e dor durante os 12 meses subsequentes ao estudo, enquanto o outro grupo manteve os resultados durante apenas os 6 meses subsequentes.

Seguindo este mesmo contexto Earl, et al., (2018), dividiram 199 participantes em dois grupos, um que realizavam exercícios para fortalecimento do quadril e quadríceps e o outro grupo executava exercícios apenas para o quadríceps, a intervenção durou seis semanas. Nem todos os participantes obtiveram êxito, sendo que apenas 89 indivíduos do grupo 1 obtiveram resultado e do grupo 2 apenas 68 indivíduos foram bem-sucedidos. Neste estudo, os resultados foram excelentes, perpetuando os resultados em quem se manteve ativo após a intervenção durando a cerca de 24 meses, havendo em alguns casos a recorrência de sintomas de apenas 5,10\% nos 24 meses pós-intervenção.

Outro fator importante também a ser relevado é o valgo dinâmico considerado um dos motivos de incidência de PFPS. Saad, et al., (2018), apontaram que exercícios de fortalecimento de joelho e quadril diminuem consideravelmente a incidência de valgo dinâmico durante atividades de abaixamento, no entanto a amostra de Villafañe, et al., (2019), identificaram melhora na dor em pacientes que receberam fortalecimento do quadril e joelho e constataram que a dor era independente à alteração do ângulo Q.

Dentro destas perspectivas e indagações sobre o trabalho de fortalecimento do quadril ou quadríceps, Hamada, et al., (2017), dividiram dois grupos com 15 pacientes em que um grupo iniciava o tratamento de quatro semanas com exercícios focando o joelho e outro grupo que realizava durante quatro semanas exercícios para os músculos do quadril. Após estas quatro primeiras semanas os grupos trocavam de protocolo constatando que no grupo que iniciou o tratamento com exercícios de quadril houve melhoras no desempenho, mais do que em atividades funcionais, enquanto os indivíduos que iniciaram o tratamento com exercícios de fortalecimento de joelho tiveram melhora maior nas atividades funcionais mais do que no desempenho e ambos os grupos obtiveram melhora na dor. 
Por sua vez Motealleh, et al., (2019), avaliaram o que seria mais eficiente no processo de tratamento, adotando o tratamento de fisioterapia de rotina ou tratamento de fisioterapia de rotina mais treinamento neuromuscular. Os grupos obtiveram êxito no tratamento embora o grupo que realizou a fisioterapia conciliando com o treinamento neuromuscular obteve resultados superiores ao grupo que realizou somente a fisioterapia.

\section{Considerações Finais}

Dentre as análises, percebe-se que a discussão sobre o tema abrange muito mais do que apenas quais exercícios devem ser usados, mas sim, qual grupamento muscular deveria ser prioridade no tratamento, sendo identificador que o fortalecimento do conjunto da musculatura do quadril e joelho (quadríceps), apresentam-se mais eficientes no tratamento da dor e melhora da funcionalidade, assim, como tratamentos fisioterapêuticos associados ao treinamento neuromuscular respeitando os limites de cada indivíduo progredindo carga ou resistência, assim que possível.

Outro fator bastante relevante é que indivíduos que executaram todos os protocolos de fortalecimento e reabilitação ao ponto de não sentir mais dor, aumentando a força e por consequência a estabilidade do joelho e se mantiveram ativos após as intervenções, foram poucos os que voltaram a se queixar de dor, percebe-se que indivíduos que se mantêm ativos prolongam os resultados alcançados durante a fase de tratamento.

\section{Referências}

Amis, A. A. (2007). Current concepts on anatomy and biomechanics of patellar stability. Sports medicine and arthroscopy review, 15(2), 48-56.

Arthur, J. R., Haglin, J. M., Makovicka, J. L., \& Chhabra, A. (2020). Anatomy and Biomechanics of the Posterior Cruciate Ligament and Their Surgical Implications. Sports Medicine and Arthroscopy Review, 28(1), e1-e10.

Baldon, R. D. M., Serrão, F. V., Scattone Silva, R., \& Piva, S. R. (2014). Effects of functional stabilization training on pain, function, and lower extremity biomechanics in women with patellofemoral pain: a randomized clinical trial. Journal of orthopaedic \& sports physical therapy, 44(4), 240-251.

Cohen, M., Ferretti, M., Marcondes, F. B., Amaro, J. T., \& Ejnisman, B. (2008). Tendinopatia patelar. Revista Brasileira de Ortopedia, $43(8), 309-318$.

Dolak, K. L., Silkman, C., McKeon, J. M., Hosey, R. G., Lattermann, C., \& Uhl, T. L. (2011). Hip strengthening prior to functional exercises reduces pain sooner than quadriceps strengthening in females with patellofemoral pain syndrome: a randomized clinical trial. journal of orthopaedic \& sports physical therapy, 41(8), 560-570.

Dye, S. F. (2005). The pathophysiology of patellofemoral pain: a tissue homeostasis perspective. Clinical Orthopaedics and Related Research $\AA_{\text {, }} 436$, 100110 .

Estrela, C. (2018). Metodologia Científica: Ciência, Ensino, Pesquisa. Editora Artes Médicas.

Earl-Boehm, J. E., Bolgla, L. A., Emory, C., Hamstra-Wright, K. L., Tarima, S., \& Ferber, R. (2018). Treatment success of hip and core or knee strengthening for patellofemoral pain: development of clinical prediction rules. Journal of athletic training, 53(6), 545-552.

Ferber, R., Bolgla, L., Earl-Boehm, J. E., Emery, C., \& Hamstra-Wright, K. (2015). Strengthening of the hip and core versus knee muscles for the treatment of patellofemoral pain: a multicenter randomized controlled trial. Journal of athletic training, 50(4), 366-377.

Flandry, F., \& Hommel, G. (2011). Normal anatomy and biomechanics of the knee. Sports medicine and arthroscopy review, 19(2), 82-92.

Ferber, R., Bolgla, L., Earl-Boehm, J. E., Emery, C., \& Hamstra-Wright, K. (2015). Strengthening of the hip and core versus knee muscles for the treatment of patellofemoral pain: a multicenter randomized controlled trial. Journal of athletic training, 50(4), 366-377.

Fukuda, T. Y., Melo, W. P., Zaffalon, B. M., Rossetto, F. M., Magalhães, E., Bryk, F. F., \& Martin, R. L. (2012). Hip posterolateral musculature strengthening in sedentary women with patellofemoral pain syndrome: a randomized controlled clinical trial with 1-year follow-up. Journal of orthopaedic \& sports physical therapy, 42(10), 823-830.

Gaitonde, D. Y., Ericksen, A., \& Robbins, R. C. (2019). Patellofemoral Pain Syndrome. American Family Physician, 99(2), 88-94.

Glaviano, N. R., \& Saliba, S. (2018). Association of altered frontal plane kinematics and physical activity levels in females with patellofemoral pain. Gait \& posture, 65, 86-88.

Glaviano, N. R., Kew, M., Hart, J. M., \& Saliba, S. (2015). Demographic and epidemiological trends in patellofemoral pain. International journal of sports physical therapy, 10(3), 281.

Grob, K., Manestar, M., Filgueira, L., Kuster, M. S., Gilbey, H., \& Ackland, T. (2018). The interaction between the vastus medialis and vastus intermedius and its influence on the extensor apparatus of the knee joint. Knee Surgery, Sports Traumatology, Arthroscopy, 26(3), 727-738. 
Research, Society and Development, v. 10, n. 9, e22110917983, 2021

(CC BY 4.0) | ISSN 2525-3409 | DOI: http://dx.doi.org/10.33448/rsd-v10i9.17983

Gulati, A., McElrath, C., Wadhwa, V., Shah, J. P., \& Chhabra, A. (2018). Current clinical, radiological and treatment perspectives of patellofemoral pain syndrome. The British Journal of Radiology, 91(1086), 20170456.

Hamada, H. A., Draz, A. H., Koura, G. M., \& Saab, I. M. (2017). Carryover effect of hip and knee exercises program on functional performance in individuals with patellofemoral pain syndrome. Journal of Physical Therapy Science, 29(8), 1341-1347.

Khayam bashi, K., Fallah, A., Movahedi, A., Bagwell, J., \& Powers, C. (2014). Posterolateral hip muscle strengthening versus quadriceps strengthening for patellofemoral pain: a comparative control trial. Archives of physical medicine and rehabilitation, 95(5), 900-907.

Makris, E. A., Hadidi, P., \& Athanasiou, K. A. (2011). The knee meniscus: structure-function, pathophysiology, current repair techniques, and prospects for regeneration. Biomaterials, 32(30), 7411-7431.

Motealleh, A., Mohamadi, M., Moghadam, M. B., Nejati, N., Arjang, N., \& Ebrahimi, N. (2019). Effects of core neuromuscular training on pain, balance, and functional performance in women with patellofemoral pain syndrome: a clinical trial. Journal of chiropractic medicine, 18(1), 9-18.

Petersen, W., Ellermann, A., Gösele-Koppenburg, A., Best, R., Rembitzki, I. V., Brüggemann, G. P., \& Liebau, C. (2014). Patellofemoral pain syndrome. Knee surgery, sports traumatology, arthroscopy, 22(10), 2264-2274.

Piazza, L. (2012). Sintomas e limitações funcionais de pacientes com síndrome da dor patelofemoral. Rev. dor, 50-54.

Piazza, L., Luza, M., Vidmar, M. F., Tavares, G. M. S., Pimentel, G. L., \& Santos, G. M. (2011). Sujeitos com Síndrome da Dor Patelofemoral apresentam fraqueza muscular e menores cargas plantares-Relato de Caso. ConScientiae Saúde, 10(3), 547-554.

Putz, r.; pabst, r. Sobotta, Atlas de anatomia humana. Guanabara Koogan, 2009.

Saad, M. C., de Vasconcelos, R. A., de Oliveira Mancinelli, L. V., de Barros Munno, M. S., Liporaci, R. F., \& Grossi, D. B. (2018). Is hip strengthening the best treatment option for females with patellofemoral pain? A randomized controlled trial of three different types of exercises. Brazilian journal of physical therapy, 22(5), 408-416.

Saltychev, M., Dutton, R. A., Laimi, K., Beaupre, G. S., Virolainen, P., \& Fredericson, M. (2018). Effectiveness of conservative treatment for patellofemoral pain syndrome: A systematic review and meta-analysis. Journal of Rehabilitation Medicine, 50(5), 393-401.

Villafañe, J. H., Bissolotti, L., La Touche, R., Pedersini, P., \& Negrini, S. (2019). Effect of muscle strengthening on perceived pain and static knee angles in young subjects with patellofemoral pain syndrome. Journal of Exercise Rehabilitation, 15(3), 454.

Vora, M., Curry, E., Chipman, A., Matzkin, E., \& Li, X. (2017). Patellofemoral pain syndrome in female athletes: A review of diagnoses, etiology and treatment options. Orthopedic reviews, 9(4).

Zamboti, C. L., Silva Junior, R. A. D., Gobbi, C., Shigaki, L., \& Macedo, C. D. S. G. (2017). Analysis of pain, functional capacity, muscular strength and balance in young women with Patellofemoral Pain Syndrome. Fisioterapia em Movimento, 30(3), 433-441. 